\title{
Primary Breast Diffuse Large B-Cell Lymphoma in a 42-Year- Old Female: A Case Report and Review of Literature
}

\author{
Maolin Chen, Jun Zhou ${ }^{\mathrm{a}}$ Xincai Qu ${ }^{\mathrm{a}, \mathrm{b}}$
}

\begin{abstract}
Primary breast diffuse large B-cell lymphoma (PB-DLBCL) is a rare localized extranodal lymphoma. It is mainly diagnosed by pathological examination due to the lack of specific clinical and imaging manifestations. Whole-body positron emission tomography-computed tomography (PET-CT) is widely used in determining clinical staging and guiding clinical treatment. As part of comprehensive treatment, targeted therapy with rituximab, intrathecal methotrexate injection and consolidation radiotherapy remain controversial in treating PB-DLBCL, but the comprehensive treatment based on full-course of chemotherapy is still widely used as the first-line treatment. Comprehensive treatment often leads to a sharp decline in the immunity of elderly patients with malignancy. In this situation, surgery may be a good chance to improve their life quality without serious complications. We present a rare case of PB-DLBCL during the coronavirus disease 2019 (COVID-19) pandemic. The patient underwent chest CT scan to screen COVID-19 and a mass of left breast was accidentally found. Because of the city lockdown policy in Wuhan, she did not seek medical help until noticing that the mass was gradually enlarged. Both ultrasonography and mammography indicated that the lesion was breast cancer. However, ultrasound-guided core needle biopsy revealed diffuse large B-cell lymphoma of breast and PET-CT scan showed that the lesion was a primary hypermetabolic tumor of left breast. The patient subsequently received comprehensive treatment based on six cycles of rituximab-cyclophosphamide, hydroxydaunomycin, oncovin, prednisone (R-CHOP) chemotherapy.
\end{abstract}

Keywords: Primary breast lymphoma; Primary breast diffuse large B-cell lymphoma; Core needle biopsy; Comprehensive treatment

\section{Introduction}

Primary breast lymphoma (PBL) is a rare form of localized

Manuscript submitted January 25, 2021, accepted February 8, 2021

Published online March 5, 2021

aDepartment of Breast and Thyroid Surgery, Union Hospital, Tongji College, Huazhong University of Science and Technology, Wuhan 430000, China ${ }^{\mathrm{b}}$ Corresponding Author: Xincai Qu, Department of Breast and Thyroid Surgery, Union Hospital, Tongji College, Huazhong University of Science and Technology, No. 1277 Jiefang Avenue, Wuhan 430000, China.

Email:whuh2018@126.com

doi: https://doi.org/10.14740/jmc3660 extranodal lymphoma without specific clinical and imaging manifestations. It constitutes $0.38-0.70 \%$ of non-Hodgkin lymphoma [1]. Primary breast diffuse large B-cell lymphoma (PB-DLBCL), which is the main pathological type of PBL, has a high degree of malignancy [2]. Through analysis of the diagnosis and treatment of a PB-DLBCL patient and review of related literature, authors hope this article will raise the understanding of the disease among clinicians.

\section{Case Report}

A 42-year-old woman was admitted to our hospital complaining of her incidental discovery of a left breast mass. She denied any relevant personal or family history. Due to the screening of coronavirus disease 2019 (COVID-19), she underwent pulmonary computed tomography (CT) examination at local hospital, which showed a $25 \times 22 \mathrm{~mm}$ nodule of the left breast. The outbreak of COVID-19 caused her failure to seek medical attention in time. Feeling that the mass was progressively enlarged and fearing that the tumor might be malignant, the patient went to our department for medical help.

Palpation examination revealed an irregular mass with medium texture behind medial side of left nipple, measuring about $40 \mathrm{~mm}$ in size. Ultrasonography showed an irregular hypoechoic nodule of approximately $44 \times 27 \mathrm{~mm}$ in size with multiple punctate calcifications in the left areola area (breast imaging reporting and database system score (BI-RADS) 4c) (Fig. 1). Mammography showed a high-density mass with partially obscured margins on inner side of the left nipple (BIRADS 4c) (Fig. 2). Based on above examination results, the diagnosis of breast cancer was preliminarily considered and radical mastectomy was put on schedule. Pathological report from ultrasound-guided core needle biopsy before operation revealed the lesion diffuse large B-cell lymphoma (DLBCL) (Fig. 3). The results of immunohistochemical staining were as follows: $\mathrm{CD} 20^{+}, \mathrm{BCL}^{+}, \mathrm{BCL}^{+}, \mathrm{MUM}^{+}, \mathrm{C}-\mathrm{Myc}^{+}$, $\mathrm{HGAL}^{+}, \mathrm{Ki}-67$ (+: 80\%), CD3-, CD5-, CD10-, CD21-, ${ }^{-}{ }^{-} 3^{-} 0^{-}$ , CyclinD1-, $\mathrm{PCK}^{-}, \mathrm{S}_{100}{ }^{-}, \mathrm{P}^{-}$. Epstein-Barr encoded RNA (EBER) was not detected in situ hybridization. To clarify the tumor stage, the patient underwent whole-body positron emission tomography-computed tomography (PET-CT) scan, which revealed a hypermetabolic mass of left breast (Fig. 4a). She was ultimately diagnosed as PB-DLBCL, nongerminal center B-cell type (non-GCB), Ann Arbor stage IA.

The patient was treated with six cycles of rituximab-cyclophosphamide, hydroxydaunomycin, oncovin, prednisone 

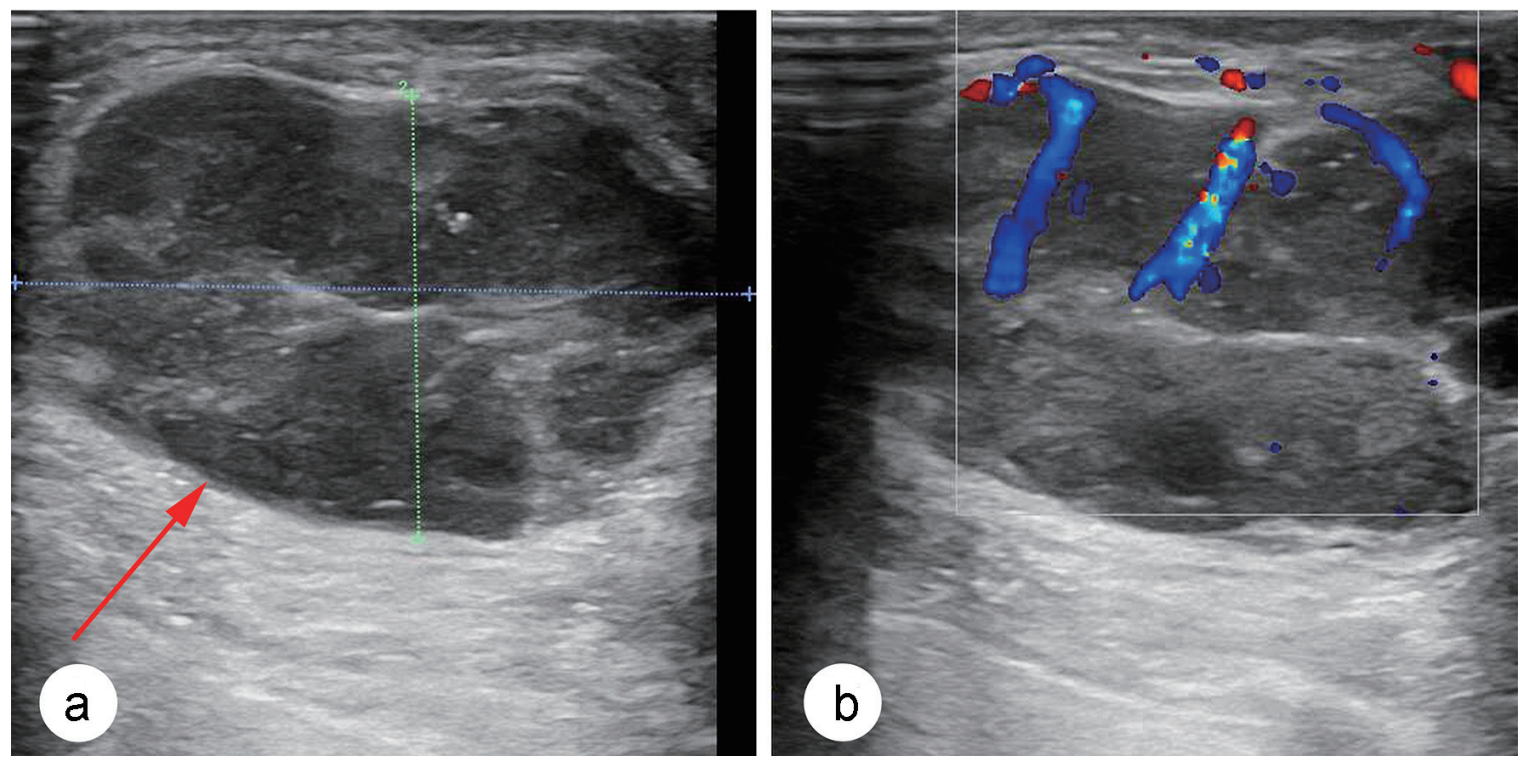

Figure 1. (a) Ultrasonography of breast showed an irregular hypoechoic nodule with multiple punctate calcifications in the left areola region (red arrow). (b) Color Doppler flow imaging revealed blood flow signals in this nodule and a high-impedance arterial flow pattern.

(R-CHOP) chemotherapy, along with five doses of intrathecal methotrexate given with the first five cycles of chemotherapy. Then two cycles of high-dose methotrexate chemotherapy and twice radiotherapy were performed. After four cycles of chemotherapy, PET-CT showed that the patient achieved complete remission (Fig. 4b). More than 6 months later, she remains in complete remission and asymptomatic.

\section{Discussion}

Primary lymphoma of breast is less common than that of other organs owing to the breast containing less lymphoid tissue [3].

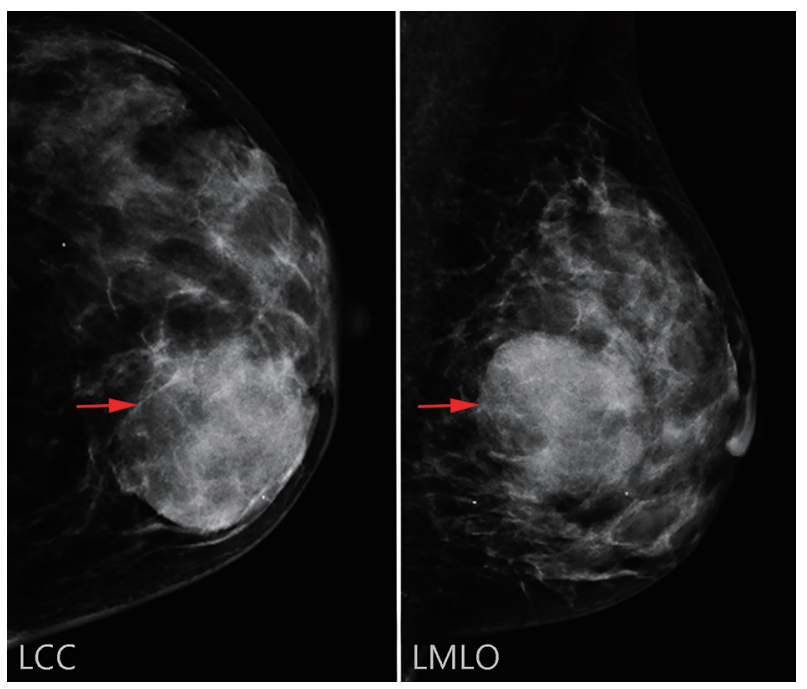

Figure 2. Mammography of left breast showed a high-density mass with partially obscured margins (red arrows).
As the main pathological type of PBL, PB-DLBCL has a high degree of malignancy $[4,5]$. It is divided into germinal center B-cell type (GCB) and non-GCB type, with the latter type having a worse prognosis [6]. Studies concerning the specific risk factors for PB-DLBCL are rare. A few studies have suggested that estrogen, chronic inflammatory diseases and autoimmune diseases may be risk factors for PBL $[7,8]$.

PB-DLBCL generally lacks specific clinical manifestations. The chief complaint of patients is usually a rapidly en-

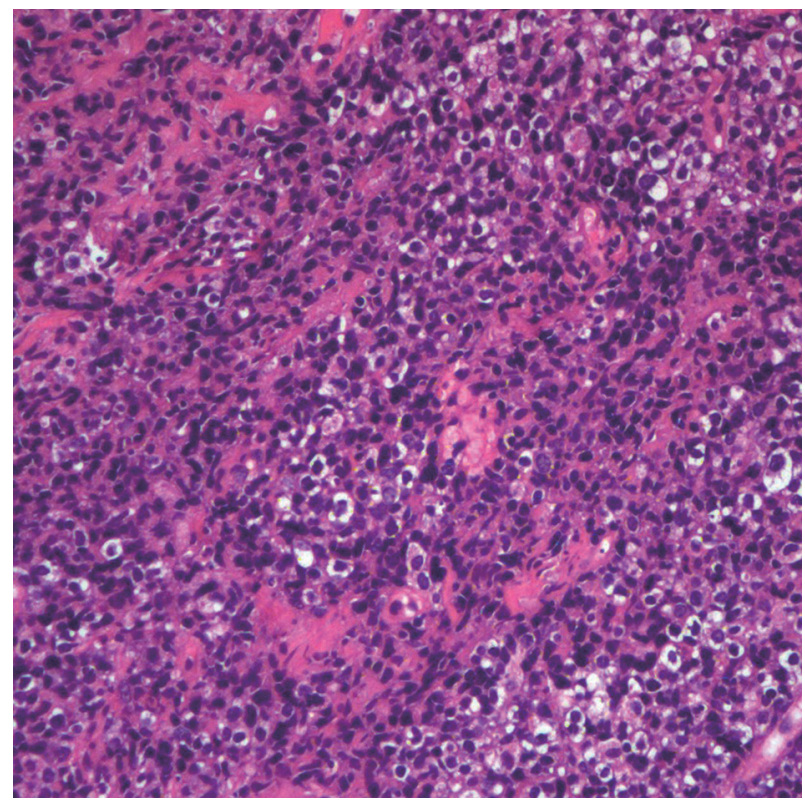

Figure 3. Frozen pathological sections revealed that the tumor cells were morphologically diverse with abundant mitotic figures (hematoxylin \& eosin (H\&E), × 100). 

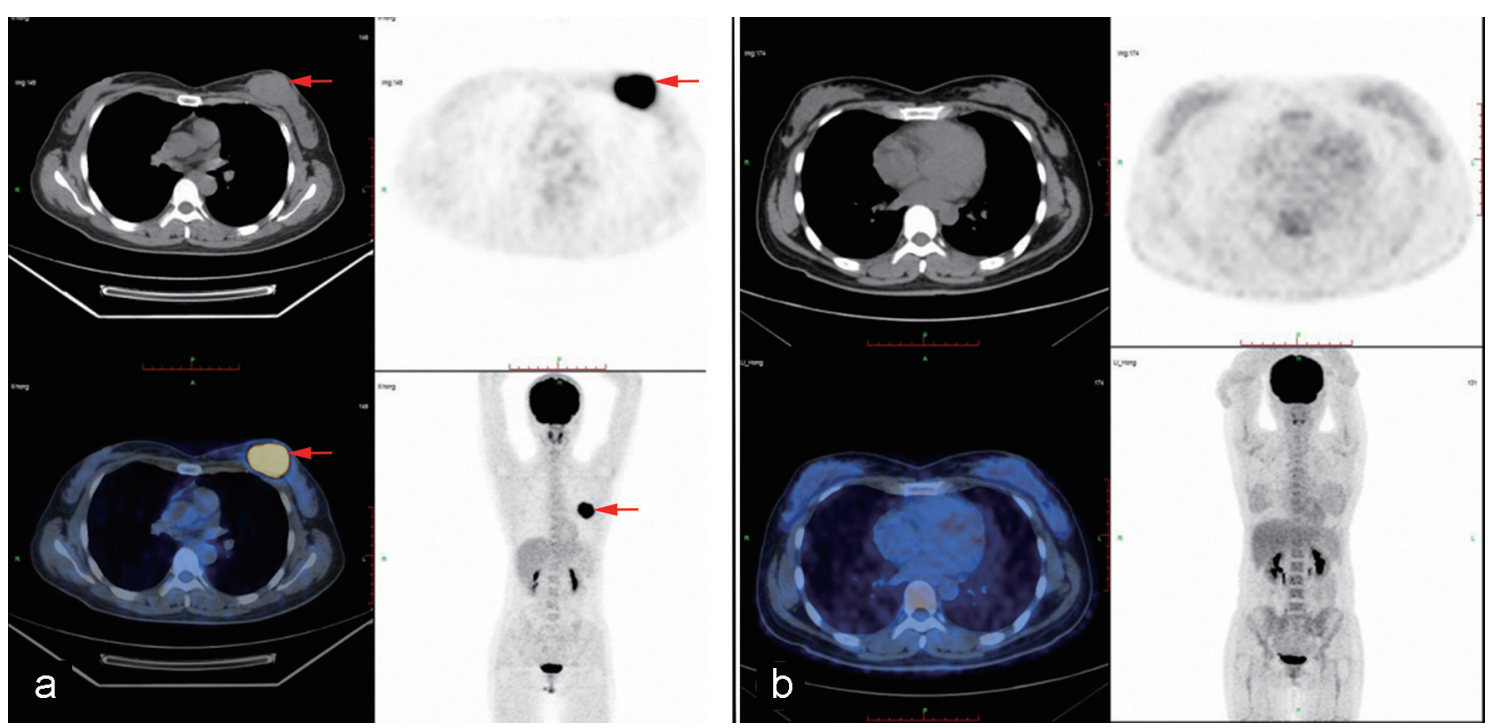

Figure 4. (a) Positron emission tomography-computed tomography (PET-CT) revealed an abnormally hypermetabolic mass of left breast (red arrows). (b) The hypermetabolic mass disappeared after four cycles of chemotherapy.

larged painless lump of breast, which is mostly seen in the upper outer quadrant, accompanied by enlarged axillary lymph nodes $[9,10]$. Sometimes the lump becomes painful on palpation, and the skin on the affected side of breast turns blue-purple $[9,11,12]$. The discrimination in the incidence of bilateral breasts is not significant. Simultaneous involvement of both sides is extremely rare and is considered to be related to poor prognosis $[4,13]$. Although there are a few characteristic imaging findings, the diagnosis of PB-DLBCL relies mainly on pathological and immunohistochemical examination [11]. Ultrasonography of PB-DLBCL usually reveals regular shaped hypoechoic mass with no calcification inside and no attenuation of the rear echo [9, 14]. Molybdenum target X-rays rarely show adverse manifestations such as burr sign and infundibulum sign $[9,11]$. Systemic PET-CT is not only helpful in the diagnosis of PB-DLBCL, but also has high specificity in the evaluation of systemic involvement, treatment effect and recurrence of patients $[14,15]$. Due to non-specific clinical and imaging manifestations and low incidence of PB-DLBCL, this patient was initially misdiagnosed with breast cancer. Comfortingly, a core needle biopsy was performed before the prepared radical mastectomy. Ultrasound-guided core needle biopsy is a safe method with high sensitivity and specificity in the diagnosis of breast lesions [16, 17]. The advantage of preoperative biopsy is that enough tissue can be obtained for immunohistochemical examination with minimal trauma. Then clinicians can identify related molecular markers to predict the prognosis of patients before treatment and determine the best scheme for patients.

Treatment strategies for PB-DLBCL have been developing during the past decades. Chemotherapy is effective in the treatment of PB-DLBCL and studies have shown that patients suffered PB-DLBCL need full-course chemotherapy [18]. First-line treatment with cyclophosphamide, hydroxydaunomycin, oncovin, prednisone (CHOP) or R-CHOP remains the standard. Compared with $\mathrm{CHOP}$ chemotherapy alone, rituxi- mab combined with chemotherapy will improve the complete remission rate and prolong disease-free survival (DFS) and overall survival (OS) of PB-DLBCL patients, without increasing clinical toxicity $[19,20]$. The effectiveness of consolidation radiotherapy is still inconclusive [14]; however, most of the data have shown that radiotherapy after chemotherapy can reduce local recurrence and improve survival benefits of patients [20,21]. Considering high recurrence rate in the central nervous system, some patients need prophylactic intrathecal injection $[18,22]$. Since brain parenchyma recurrence is more common than leptomeninges $[13,23]$, systemic central nervous system chemotherapy such as high-dose intravenous methotrexate may be a more promising option than prophylactic intrathecal injection to prevent nervous system recurrence. Radical surgery seems to be associated with higher mortality and poorer prognosis [24]. If radical operation is performed because of misdiagnosis as breast cancer, the follow-up comprehensive treatment should be carried out as soon as possible.

Since most studies were based on relatively small sample sizes, the effectiveness of rituximab, prophylactic intrathecal injection and radiotherapy for consolidation is still controversial $[13,18,20]$. Further prospective studies are needed to demonstrate their effectiveness in patients with PB-DLBCL. In order to achieve the best therapeutic effect, the patient received comprehensive treatment based on R-CHOP chemotherapy. She has now reached complete remission. Although chemotherapy is the first-line treatment, it may cause severe immunodeficiency and even fatal complications of elderly patients [25]. In order to prevent such situations from happening, palliative surgery is a good chance to improve the life quality of patients.

\section{Conclusions}

In summary, the final diagnosis of PB-DLBCL mainly depends 
on the pathological and immunohistochemical examination. The effectiveness of consolidation radiotherapy, rituximab, prophylactic intrathecal injection and high-dose intravenous methotrexate still needs to be verified by large-scale prospective studies, but synthetic therapy based on full-course chemotherapy is still recognized as the most effective treatment of PB-DLBCL to date.

\section{Acknowledgments}

None to declare.

\section{Financial Disclosure}

None to declare.

\section{Conflict of Interest}

None to declare.

\section{Informed Consent}

Informed consent was obtained.

\section{Author Contributions}

MLC performed the case report and wrote the manuscript; JZ collected the data and revised the manuscript; XCQ performed consultation and edited the manuscript; all authors issued final approval for the version to be submitted.

\section{Data Availability}

Any inquiries regarding supporting data availability of this study should be directed to the corresponding author.

\section{References}

1. Avenia N, Sanguinetti A, Cirocchi R, Bistoni G, Trastulli S, D'Ajello F, Barberini F, et al. Primary breast lymphomas: a multicentric experience. World J Surg Oncol. 2010;8:53.

2. Jennings WC, Baker RS, Murray SS, Howard CA, Parker DE, Peabody LF, Vice HM, et al. Primary breast lymphoma: the role of mastectomy and the importance of lymph node status. Ann Surg. 2007;245(5):784-789.

3. Ferguson DJ. Intraepithelial lymphocytes and macrophages in the normal breast. Virchows Arch A Pathol Anat Histopathol. 1985;407(4):369-378.

4. Thomas A, Link BK, Altekruse S, Romitti PA, Schroeder MC. Primary Breast Lymphoma in the United States:
1975-2013. J Natl Cancer Inst. 2017;109(6).

5. Jia Y, Sun C, Liu Z, Wang W, Zhou X. Primary breast diffuse large B-cell lymphoma: a population-based study from 1975 to 2014. Oncotarget. 2018;9(3):3956-3967.

6. Lenz G, Wright G, Dave SS, Xiao W, Powell J, Zhao H, $\mathrm{Xu} \mathrm{W}$, et al. Stromal gene signatures in large-B-cell lymphomas. N Engl J Med. 2008;359(22):2313-2323.

7. Domchek SM, Hecht JL, Fleming MD, Pinkus GS, Canellos GP. Lymphomas of the breast: primary and secondary involvement. Cancer. 2002;94(1):6-13.

8. Teras LR, Patel AV, Hildebrand JS, Gapstur SM. Postmenopausal unopposed estrogen and estrogen plus progestin use and risk of non-Hodgkin lymphoma in the American Cancer Society Cancer Prevention Study-II Cohort. Leuk Lymphoma. 2013;54(4):720-725.

9. Surov A, Holzhausen HJ, Wienke A, Schmidt J, Thomssen C, Arnold D, Ruschke K, et al. Primary and secondary breast lymphoma: prevalence, clinical signs and radiological features. Br J Radiol. 2012;85(1014):e195-205.

10. Kuper-Hommel MJ, Snijder S, Janssen-Heijnen ML, Vrints LW, Kluin-Nelemans JC, Coebergh JW, Noordijk EM, et al. Treatment and survival of 38 female breast lymphomas: a population-based study with clinical and pathological reviews. Ann Hematol. 2003;82(7):397404.

11. Sabate JM, Gomez A, Torrubia S, Camins A, Roson N, De Las Heras P, Villalba-Nuno V. Lymphoma of the breast: clinical and radiologic features with pathologic correlation in 28 patients. Breast J. 2002;8(5):294-304.

12. Cheah CY, Campbell BA, Seymour JF. Primary breast lymphoma. Cancer Treat Rev. 2014;40(8):900-908.

13. Hu S, Song Y, Sun X, Su L, Zhang W, Jia J, Bai O, et al. Primary breast diffuse large B-cell lymphoma in the rituximab era: Therapeutic strategies and patterns of failure. Cancer Sci. 2018;109(12):3943-3952.

14. Raj SD, Shurafa M, Shah Z, Raj KM, Fishman MDC, Dialani VM. Primary and Secondary Breast Lymphoma: Clinical, Pathologic, and Multimodality Imaging Review. Radiographics. 2019;39(3):610-625.

15. Kong EJ, Cho IH. F-18 FDG PET/MRI findings of primary breast lymphoma in two cases: FDG PET/MRI findings of primary breast lymphoma. Clin Imaging. 2015;39(4):682-684.

16. Rakha EA, Ellis IO. An overview of assessment of prognostic and predictive factors in breast cancer needle core biopsy specimens. J Clin Pathol. 2007;60(12):1300-1306.

17. Giuliani M, Patrolecco F, Rella R, Di Giovanni SE, Infante A, Rinaldi P, Romani M, et al. Can breast cancer biopsy influence sentinel lymph node status? Clin Breast Cancer. 2016;16(6):153-157.

18. Yhim HY, Kang HJ, Choi YH, Kim SJ, Kim WS, Chae YS, Kim JS, et al. Clinical outcomes and prognostic factors in patients with breast diffuse large B cell lymphoma; Consortium for Improving Survival of Lymphoma (CISL) study. BMC Cancer. 2010;10:321.

19. Pfreundschuh M, Trumper L, Osterborg A, Pettengell R, Trneny M, Imrie K, Ma D, et al. CHOP-like chemotherapy plus rituximab versus $\mathrm{CHOP}-$ like chemotherapy alone in young patients with good-prognosis diffuse large-B- 
cell lymphoma: a randomised controlled trial by the MabThera International Trial (MInT) Group. Lancet Oncol. 2006;7(5):379-391.

20. Ryan G, Martinelli G, Kuper-Hommel M, Tsang R, Pruneri G, Yuen K, Roos D, et al. Primary diffuse large B-cell lymphoma of the breast: prognostic factors and outcomes of a study by the International Extranodal Lymphoma Study Group. Ann Oncol. 2008;19(2):233-241.

21. Liu PP, Wang KF, Jin JT, Bi XW, Sun P, Wang Y, Yang H, et al. Role of radiation therapy in primary breast diffuse large B-cell lymphoma in the Rituximab era: a SEER database analysis. Cancer Med. 2018;7(5):1845-1851.

22. Aviles A, Delgado S, Nambo MJ, Neri N, Murillo E, Cleto S. Primary breast lymphoma: results of a controlled clinical trial. Oncology. 2005;69(3):256-260.

23. Kansara R, Villa D, Gerrie AS, Klasa R, Shenkier T, Scott DW, Slack GW, et al. Site of central nervous system (CNS) relapse in patients with diffuse large B-cell lymphoma (DLBCL) by the CNS-IPI risk model. Br J Haematol. 2017;179(3):508-510.

24. Aviv A, Tadmor T, Polliack A. Primary diffuse large B-cell lymphoma of the breast: looking at pathogenesis, clinical issues and therapeutic options. Ann Oncol. 2013;24(9):2236-2244.

25. Pamoukdjian F, Liuu E, Caillet P, Herbaud S, Gisselbrecht M, Poisson J, Boudou-Rouquette P, et al. How to optimize cancer treatment in older patients: an overview of available geriatric tools. Am J Clin Oncol. 2019;42(2):109-116. 